\title{
Cytomegalovirus pp65-Specific Cytotoxic T Lymphocytes
}

National Cancer Institute

\section{Source}

National Cancer Institute. Cytomegalovirus pp65-Specific Cytotoxic T Lymphocytes. NCI

Thesaurus. Code C71747.

Cytotoxic T lymphocytes (CT Ls) specifically reactive to the cytomegalovirus (CMV) phosphoprotein pp65 with potential antiviral activity. To prepare CMV pp65-specific cytotoxic T lymphocytes in vitro, dendritic cells (DCs) are pulsed with CMV pp65 epitopes and then used to stimulate and propagate CMV pp65-specific cytotoxic T lymphocytes from peripheral blood mononuclear cells (PBMNCs); the CMV pp65-specific cytotoxic T lymphocyte population is then expanded so as to be sufficient for use in adoptive T lymphocyte therapy. When administered into a patient post-allogeneic hematopoietic stem cell transplantation, this agent may elicit a specific CT L response against CMVinfected host cells, which may result in the resolution of CMV infection. The CMV pp65 protein (65 kDa lower matrix phosphoprotein), the primary component of the enveloped subviral particle, is an immunodominant target for helper and cytotoxic T lymphocyte responses to CMV. 